\title{
SOBRE LAS REGLAS COMO RAZONES EXCLUYENTES. UN COMENTARIO AL TRABAJO DE JUAN CARLOS BAYÓN*
}

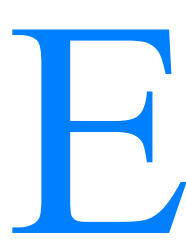

1 artículo de Bayón (y, por supuesto, su libro ${ }^{1}$ ) me parece un trabajo excelente, estimulante y bien construido. La mía es, en consecuencia, una tarea difícil; ello explica, y espero que pueda justificar, el hecho de que plantearé un conjunto de problemas más que un comentario orgánico acerca del artículo de Bayón, el cual ciertamente merece más que mis pocas palabras.

El concepto de razones para la acción, en los trabajos de $\operatorname{Raz}^{2}$ y en el lenguaje común, muestra, en mi opinión, una ambigüedad fundamental; algunos estudiosos como, por ejemplo, Carlos Nino ${ }^{3}$ y Mario Jori ${ }^{4}$, pusieron igualmente énfasis, hace algunos años, en esta ambigüedad. «Razones para la acción» denota conjuntamente y, a menudo, confusamente, tanto los motivos (generalmente hablando, las causas psicológicas de una acción intencional) 1994.

${ }^{1}$ Cfr. J.C. Bayón Mohíno, La normatividad del derecho: deber jurídico y razones para la acción, Centro de Estudios Constitucionales, Madrid, 1991.

2 Cfr. J. Raz, Razón práctica y normas $\left(1975,1990^{2}\right)$, trad. de J. Ruiz Manero, Centro de Estudios Constitucionales, Madrid, 1990; Id. The Authority of Norms. Essays on Law and Morality, Oxford, Clarendon Press, 1979; Id., The Morality of Freedom, Oxford, Oxford University Press, 1986 (sobre el cual véase D. H. Regan, «Authority and Value: Reflections on Raz's Morality of Freedom», en Southern California Law Review, 62, 3-4, 1989, pp. 995-1095); Id., «Facing up: A Reply» en Southern California Law Review, 62, 3-4, 1989, pp. 1153-1235.

${ }^{3}$ Cfr. C.S. Nino, La validez del derecho, Astrea, Buenos Aires, 1985; Id., Introducción a la filosofía de la acción humana, EUDEBA, Buenos Aires, 1987.

${ }^{4}$ Cfr. M. Jori, «Nuovi sviluppi in teoria delle norme», en Sociologia del diritto, 1, 1979, pp. 171-177; Id., Il formalismo giuridico, Giuffrè, Milano, 1980. Sobre el concepto de razones para actuar, véanse también: R. Caracciolo, «El concepto de autoridad normativa. El modelo de las razones para la acción», en Doxa, 10, 1991, pp. 67-90; P.E. Navarro-M.C. Redondo, «Legal Norms and Practical Resoning, A Critique of Joseph Raz», en Rechtstheorie, 23, 1992, pp. 491-498; M.C. Redondo, Normas jurídicas, aceptación y justificación. La noción de razón para la acción como instrumento de análisis conceptual, tesis doctoral (Universidad Pompeu Fabra, Barcelona, año 1994-95). 
como las premisas del razonamiento práctico que justifican una acción. Creo que se pueden encontrar, en Raz, las siguientes dificultades:

a) no se distingue claramente entre esos dos sentidos de «razones para la acción»: tienen, por supuesto, relación mutua, pero, desde un punto de vista conceptual, no deberíamos confundirlos;

b) no se distingue claramente entre explicación y justificación (quizás, en general, por la falta de distinción en la obra de Raz entre enunciados descriptivos y enunciados normativos);

c) no se distingue claramente entre análisis lingüístico (y, dentro de él, entre inventariar y estipular el sentido de las palabras), análisis conceptual e investigación psicológica o conductista.

Mi objetivo, aquí, no es criticar directamente a Raz: Bayón ya lo ha hecho muy bien. No debemos olvidar, sin embargo, que necesitamos distinguir con cuidado, lo que Raz deja de hacer a menudo, al menos entre dos usos de «razones para la acción»: uno explicativo y otro justificativo.

Bayón también enfatiza correctamente el problema del papel de las razones para la acción en la teoría de la decisión racional. Especialmente, en su artículo, destaca el problema de si seguir una regla, como razón excluyente para la acción, podría o no ser un modelo para tomar decisiones racionales.

Presentaré algunas observaciones relativas a los tres usos potenciales de «reglas como razones excluyentes»: en la explicación del razonamiento jurídico; en la justificación de una conclusión en el razonamiento jurídico; en la teoría de la decisión racional en el ámbito jurídico.

La base (parcialmente) común de mis observaciones y preguntas es la siguiente. Bayón probablemente está en lo cierto cuando, interpretando la obra de Raz, le atribuye una concepción cerrada de razones excluyentes, y la critica como normativamente inconsistente y carente de rendimiento explicativo. En la interpretación de Bayón de las teorías de Raz y Schauer ${ }^{5}$, las reglas podrían suministrar razones excluyentes sólo si son concebidas como provistas de autonomía semántica, es decir, como reglas en sentido estricto. Además, habría una conexión necesaria entre esas reglas como razones excluyentes y el llamado modelo basado en reglas (Rule-Based Model): sólo dentro del modelo basado en reglas del razonamiento jurídico, las reglas podrían funcionar como razones excluyentes. Si aceptamos esta interpretación de la tesis de Raz, que, como ya he dicho, probablemente es la mejor, es necesario abandonar la intuición de Raz sobre el papel central de las reglas, como razones excluyentes, en el razonamiento jurídico. Antes de aceptar este

${ }^{5}$ Cfr. F. Schauer, Playing by the Rules. A Philosophical Examination of Rule-Based Decision Making in Law and Life, Oxford, Clarendon Press, 1991. 
resultado, sería mejor, en mi opinión, intentar otro camino, de forma que: a) podríamos usar una concepción menos cerrada de una regla como una razón excluyente, y b) podríamos, entonces, debilitar la conexión entre las reglas como razones excluyentes y el modelo basado en reglas. Por lo que concierne al primer punto, podríamos adoptar la siguiente definición de regla como razón excluyente: una regla es un enunciado-tipo normativo, emitido por el legislador y aún sin interpretar. Esta regla deberá ser interpretada de manera que pueda ser aplicada en un caso concreto. Hay, en efecto, muchas interpretaciones semántica y jurídicamente posibles de una regla; cada una de estas interpretaciones necesita justificación jurídica. En consecuencia, en lo que concierne al segundo punto, las reglas como razones excluyentes juegan un importante papel no sólo en el modelo basado en reglas, sino también, en particular, en el modelo particularista sensible a las reglas (Rule-Sensitive Particularistic Model) del razonamiento jurídico. Intentaré explicar en lo que sigue, muy brevemente, ese uso alternativo de la intuición de Raz, mientras subrayo algunos problemas de Bayón.

1) Puede verse, en primer lugar, el uso del concepto de razones excluyentes en la explicación del actual razonamiento jurídico de los jueces, abogados y ciudadanos: un grupo de personas que, por supuesto, no son agentes completamente racionales en el sentido de la teoría de la decisión racional. Generalmente las reglas jurídicas, en su dominio, funcionan como razones excluyentes (es decir, motivos): generalmente, las personas toman decisiones, en el campo jurídico, guiadas por reglas, y no por otros tipos de razones. En particular, no se guían por las mismas razones pora las que generalmente no ponderan, no balancean esas razones, si existe una regla jurídica y prescribe una conducta determinada. Esa regla, por supuesto, debe ser interpretada por los jueces, abogados y ciudadanos, de forma que desempeñe su papel de guía. Pero no puedo abordar aquí este punto.

¿Por qué las reglas jurídicas juegan este papel? La explicación es bien conocida. Las reglas jurídicas son, a menudo, respaldadas por sanciones, el derecho hace efectivo el cumplimiento de las reglas a través de su mecanismo de realimentación (estoy usando una expresión de Olivecrona ${ }^{6}$ ), las actuales actitudes conformistas de la gente ayudan a esa maquinaria, seguir una regla es un mecanismo de ahorro de tiempo y trabajo, seguir una regla es, a menudo, el camino más fácil, etcétera.

He dicho muchas veces «generalmente». ¿Por qué?, Porque, evidentemente, algunas veces las reglas jurídicas no funcionan como razones excluyentes

${ }^{6}$ K. Olivecrona, Law as fact, London, Oxford University Press, 1939. 
(es decir, motivos) para alguna gente. En general, esto sucede cuando las reglas jurídicas se apartan demasiado de otros tipos de razones para algunos agentes. Este es el caso, por ejemplo, del que hurta (el cual tiene fuertes motivos, fuertes intereses, en contra de la regla), o del desobediente civil (el cual tiene fuertes motivos, fuertes valores morales, en contra de la regla).

Entonces, usualmente pero no siempre, las reglas funcionan como razones excluyentes. Podríamos decir, por supuesto, que sólo las normas (las diferentes interpretaciones de las reglas) juegan ese papel como motivos de las decisiones de la gente; pero, normalmente, sin la existencia de la regla, ninguna norma podría jugar ese papel. Sé muy bien, en efecto, que el problema es mucho más complejo que esto, pero, como ya he dicho, no puedo enfrentarme aquí a este punto.

2) En segundo lugar, podemos ver el uso del concepto de razón excluyente en la justificación de una conclusión en el razonamiento jurídico. Presentaré tres observaciones.

a) Por lo que concierne a la tipología de los modelos de razonamiento jurídico de Schauer, si adoptamos mi definición estipulativa de regla, podemos observar algunas analogías entre esos modelos y algunos tipos muy conocidos de argumentación interpretativa ${ }^{7}$ en el campo jurídico. Específicamente: i) El modelo particularista me parece semejante a la aplicación de una regla interpretada mediante el uso del argumento ratio legis. La argumentación teleológica se refiere a los fines, o a los objetivos, de una regla, pero no se puede ignorar completamente la existencia y la formulación del texto legal. ii) El modelo basado en reglas me parece muy rígido para la aplicación de una regla «literalmente» interpretada, de acuerdo al significado ordinario o técnico de las palabras. iii) El modelo particularista sensible a las reglas me parece semejante a la aplicación de una regla interpretada mediante el uso del argumento ratio iuris o argumento basado en los principios legales (normalmente constitucionales). En cada modelo, las reglas son razones excluyentes en este sentido específico: su existencia excluye toda referencia a cualquier razón fuera del dominio jurídico. En el tercer modelo, en particular, encontramos referencia a otras razones; pero esas razones son otras reglas (o interpretaciones de reglas), jerárquicamente superiores, que priman en ese caso sobre la regla interpretada. Podríamos decir, en ese caso, por ejemplo, que algunas reglas constitucionales funcionan como reglas excluyentes.

b) En lo referente a la tesis principal de Bayón («un agente racional no puede en ningún caso aceptar reglas como razones excluyentes»), estoy de

${ }^{7}$ Cfr. Tarello, L'interpretazione della legge, Milano, Giuffrè, 1980. 
acuerdo con él si se reduce el alcance de su tesis, diciendo: «un agente racional no debería, en elámbito jurídico, aceptar siempre reglas jurídicas como razones excluyentes». Esta formulación garantiza un espacio, por ejemplo, para la desobediencia civil: mis preferencias morales también van en esa dirección. No creo, sin embargo, que Bayón esté de acuerdo conmigo en la reducción del alcance de su tesis. Algunas observaciones y preguntas son necesarias.

I) La tesis principal de Bayón, incluso si fuese correcta, me parece bastante tautológica. Según la opinión de Bayón, una agente racional es aquel que actúa en concordancia con lo que debe hacer, y él debe hacer lo que, considerando todas las cosas, tiene que hacer. Entonces, hacer, o tomar una decisión, considerando todas las cosas significa actuar sobre la base del balance de todas las razones relevantes. En efecto, actuar sobre la base de una razón excluyente no es actuar considerando todas las cosas, y consecuentemente, por mera definición, no es actuar racionalmente.

II) ¿Cuál es el significado de «racional» en el paper de Bayón? Algunas veces parece hacer referencia a una concepción instrumental de racionalidad; otras veces, parece deslizarse hacia una concepción substantiva de la racionalidad. Pero, quizás, en esos últimos casos no he interpretado correctamente su pensamiento.

III) ¿Por qué no deberíamos ser «irracionales»? O, en otras palabras, ¿por qué deberíamos aceptar la definición de «racionalidad» de Bayón? Creo que es imposible encontrar una fundamentación moral última, y también una fundamentación moral última de la racionalidad. Dos contraejemplos a la principal tesis de Bayón son relevantes para mi opinión. III') Legalismo moral: las reglas jurídicas son razones (morales) excluyentes para la acción. No estoy de acuerdo con el legalismo moral y, seguramente, Bayón tampoco. Pero es una elección moral última sin fundamentación última como la racionalidad de Bayón. III') Deontologismo moral: un sistema de reglas o principios morales, jerárquicamente ordenados, que son todos razones excluyentes de diferente grado. Estoy de acuerdo con el deontologismo moral (una versión del principio $\mathrm{P}_{3}$ de Raz: «Es siempre el caso que se debe, todas las cosas consideradas, actuar por una razón no derrotada $\rangle^{8}$ y seguramente Bayón no compartirá mi opinión. Pero, una vez más, ésta es una elección moral última, sin ninguna justificación complementaria. Advierto, de paso, que, en mi interpretación de $\mathrm{P}_{3}$, «todas las cosas consideradas» significa que tenemos que considerar, en cada caso, todas las reglas o principios del sistema moral que aceptamos.

${ }^{8}$ J. Raz, Razón práctica y normas, cit. p. 45. 
c) Finalmente, en lo que concierne a la teoría de la decisión racional en el campo jurídico, propondría dos comentarios más.

El primero es muy ingenuo o, si se prefiere, de sentido común. Si consideramos la principal tesis de Bayón como un criterio para tomar decisiones racionales (y no como un imperativo moral, al igual que anteriormente), ese criterio, en la vida actual, nos llevará probablemente a la muerte, como al asno de Buridán. No podemos decidir siempre sobre la base de todas las razones relevantes, simplemente porque no tenemos tiempo suficiente.

El segundo es, en positivo, la otra cara de la misma moneda. Como algunos teóricos de la decisión racional han mostrado, el utilitarismo de la regla es probablemente una mejor alternativa que el utilitarismo del acto. Puede decirse: considerando todas las cosas tenemos que elegir el utilitarismo de la regla y no el del acto. Después de todo, tenemos que actuar de acuerdo a la elección de reglas como razones excluyentes.

(Trad. de Jordi Ferrer) 